\title{
A Tool for Systematically Accessing the Level of Readiness of Engineering Design in Product Development
}

\author{
K. Behdinan ${ }^{1}$, M. Fahimian ${ }^{1}$, R. Pop-Iliev ${ }^{2}$. \\ ${ }^{l}$ Department of Mechanical and Industrial Engineering, University of Toronto. \\ ${ }^{2}$ Faculty of Engineering and Applied Science, University of Ontario Institute of Technology. \\ * mahi.fahimian@utoronto.ca
}

\begin{abstract}
This paper introduces a top down, systemengineering approach to develop a quantifiable and systematic tool, referred to as Design Readiness Level (DRL), to gauge design at each stage of product development. It is developed to facilitate communication between different stockholders of a design project and to address the complexities arising during all the phases of product design, from initiation to completion. The design process as one of the pillars of DRL has been studied thoroughly and is categorized into nine stages to reflect the technical flow in product development. The design stages are iterative at any level from 1 to 9 and have distinctive deliverables at the end of each stage. The deliverables simplify and characterize the assessment of the design from the technical point of view. Developing a comprehensive DRL metrics that encompasses all the stockholders' perspectives in a design is a work in progress.
\end{abstract}

Keywords: Design Readiness Level, Design Management, Multidisciplinary Engineering Design, Product Development, Design Assessment.

\section{INTRODUCTION}

Design engineering includes an extensive and comprehensive transformation process from customer needs to an actual useful product. A design problem as the trigger of the design can be presented in multiple forms: from a rough sketch of an ill-defined problem with little information, to a more detailed problem of application of a patent for different working or manufacturing environment or translation of scientific research findings to a practical application. The design domain can also vary extensively, it can make use of an already developed technology or it might develop a new technology within its domain of development [1]. The huge variability in initiation, the level of analysis and the domain of a particular design, makes the assessment of its level a very complicated task. The accomplishment of this task is significantly aggravated when attempting to assess multidisciplinary engineering design projects, at which the need for an objective and repeatable assessment method is most emphasized. Therefore, managing and evaluating the large amount of information from multiple sources in a multidisciplinary design at the stage of gaining support from external stakeholders and allocating resources in an academic setting, or during the acquisition phase in industry, needs a systematic metric tool that can bridge the gap between business and technical stakeholders and support the decision-making process.

Readiness level ontology metrics have been developed for technology and manufacturing capabilities [2, 3]. Technology Readiness level (TRL) has been developed by NASA to gauge the development of different space technologies [2]. TRL consists of nine stages benchmarking a technology development from basic scientific principle up to an actual system proved through successful mission operation. NASA's most up-to-date TRL documentation is publicly available in their systemengineering handbook [4].

The detailed definitions of TRL with terminology descriptions are published by Airforce Research laboratory for national fixed wing vehicle program [5], however the definitions were specific for aeronautical technologies. To help project managers in applying TRL as a decision making tool [6], Moorhouse [5] has given an appropriate risk level to each TRL, however, the risk level definitions were qualitative and nonspecific. TRL assessment is focused on low volume space technologies . Starting in early nineties, the TRL system was adopted by different industrial sectors. Its application was mostly to manage and monitor the risk of acquisition of technologies in different stages of development [7, 8]. However, the adaptation of the TRL in different industries, encountered challenges in terms of its integration and connectivity, scope of TRL assessment, product road mapping or imprecision of the scales $[9,10]$. 
On the other hand, design in terms of its scope and evolutionary process, is different from technology. The design process covers a larger ground than technology because challenges such as integration of technology components, manufacturability, or environment readiness need to be addressed as well [11]. The usefulness of a design assessment tool becomes more critical as so many industries such as Apple, Philips, Nokia or Sony create competitive advantages through their innovative designs [12].

A comprehensive assessment tool for design is needed to be able to benchmark and effectively evaluate the cost and risk of a design at each step of its development or acquisition [13, 14]. It could be effectively used as a communication tool between managerial decision makers, investors, and technical people. In order to develop a hierarchical Design Readiness (DR) metrics as a systematic tool to access the level of readiness of design engineering efforts in product development, a holistic approach of problem solving needs to be adopted.

\section{DESIGN STAGES}

In this context, the first step would include analyzing the design from multiple perspectives. One perspective is the technical perspective of the process of design. This perspective looks at necessary stages that each design problem needs to go through to transform to a product that can satisfy the customer needs. This paper introduces the Design Readiness Level (DRL) and is focused on the design process as one pillar of DRL. In following section the design process has been categorized into nine distinctive stages with its own deliverables.

Each design project needs to go through a process in order to transform a need, an idea or a scientific finding to a useful product or system that can be marketed. The goal of the design process is not to restrict the innovation or eliminate changes but to manage the evolution of the design so that most changes come through iterations early in the process. Common iterative phases in the design engineering process could be categorized in nine Design Stages (DS):

DS 1: Design Team(s) Commensurate to the Project Idea and Scope Established. In this stage of the design, the theoretical background of the design problem is well established and the project related current state of art is reviewed. The result of this stage is that the required expertise needed for the design team(s) is established and the design team(s) with required expertise are formed.
DS 2: The Design Problem is Well Understood. In this stage the design problem is defined based on customer needs. In addition, the envisioned initial project scope is expanded and the boundary of the design problem is defined. The result of this stage is the translation of customer needs and project scope to precise engineering language that includes measurable and comparable values and limitations.

DS 3: Concurrent/Simultaneous/ Integrated Design Approach Implemented. At this stage, the recourses necessary for accomplishing the design are recognized and the detail description of proposed approaches to solve the design problem are studied. As a result, a detailed project plan, documenting the design activities, roles, timelines and deliverables are established. Importantly, this stage is characterized by conducting the design of the product and the design of its production process simultaneously.

DS 4: Proof of Concept Prototype Developed and Tested. At this stage of the design, engineering methods and tools to solve the problem are identified. Additionally, feasible design concepts are generated and evaluated. At the end of this stage a proof of concept of selected solutions that could be a physical artifact or simulation is generated and tested.

DS 5: Proof-of-Product Prototype Developed and Tested. At this stage of the design, the form and shape of the embodiments of the product design parameters is established through industrial and haptic design. In addition, end of life and economic analysis is done. The result of this stage is a proof of product prototype that is built and tested.

DS 6: Proof-of-Production Prototyping Completed. At this stage of the design, the proof of product prototype is documented and failure mode and effects (FMEA) analysis is done. In addition, all the instructions regarding the assembly, installation, maintenance, recycling of the product is documented through a product manual.

DS 7: Final Design Refinements Implemented. This stage of the design is for final revisions that could be the result of evaluating the issues and inconsistencies of performance during any proof-of prototype stage, or it can come from the feedback received from customers or other project stakeholders. The changes are documented and if necessary other design stages are repeated to incorporate the necessary changes. 


\section{DS 8: Product Validation and Verification}

Completed. This stage includes developing a testing plan for the product based on the current test standards or a newly developed testing method. The testing plan and the test result might impose more changes in the design that need to be addressed in this stage.

DS 9: Capability to launch the product achieved. This stage could include creating a pilot production line to establish the manufacturability of the product. At this stage the product is launched and is handed over to the customers. This stage could include a post launch feedback analysis.

The design stages presented above are evolutionary as later stages are more advanced compared to initial stages, however, in order to meet the required deliverables at a specific stage, multiple iterations might need to be undertaken.

\section{CONCLUSIONS}

In order to evaluate a multidisciplinary engineering design project at each stage of its development, for the purpose of further investment, academic evaluation or allocating resources in academia, a systematic and comprehensive assessment tool namely Design Readiness Leve (DRL) is needed. DRL will act as a communication tool between technical and business or academic decision makers. To develop a holistic tool, design needs to be evaluated through multiple perspectives based on the needs and practice of different stakeholders. In this paper one perspective, which is the technical perspective, is studied and the design process is categorized into nine stages. Each stage of the design process has a distinct and measurable outcome which simplifies evaluating the design process in terms of its deliverables. The development of other perspectives of design is still in progress.

\section{References}

[1] Morteza Poorkiany, Joel Johansson Fredrik Elgh, "Capturing, structuring and accessing design rationale in integrated product design and manufacturing processes," Advanced Engineering Infomatics, vol. 30, pp. 522-536, 2016.Available as of August, 2016 from http://dx.doi.org/10.1016/j.aei.2016.06.004

[2] C.J. Mankins, "Technology Readiness Level, White Paper", Advanced Concepts Office of Space Access and Technology NASA, April 1995.
[3] M. J. Ward, S. T Halliday and J Foden, “A readiness level approach to manufacturing technology development in the aerospace sector: An industrial approach", Proc. I. MechE Vol 226, Part B, pp. 547-552.

[4] NASA. NASA Systems Engineering Handbook, Washington, D.C, 2007.

[5] David J. Moorhouse, "Detailed Definitions and Guidance for Application of Technology Readiness Levels", J. Aircraft, Vol39, No. 1: pp. 190-192.

[6] Robert G. Cooper, "Managing technology development projects," Industrial Research Institute Inc., pp. 23-31, November- December 2006.

[7] European Association of Research and Technology Organizations, "The TRL Scale as a Research \& Innovation Policy Tool, EARTO Recommendations”, 2014.

[8] J. Rybicka, A.Tiwari, and G.A. Leeke, "Technology readiness level assessment of composites recycling technologies", Journal of Cleaner Production, 112, pp.1001-1012.

http://dx.doi.org/10.1016/j.jclepro.2015.08.104

[9] A. Olechowski, S.D. Eppinger and N. Joglekar, "Technology Readiness Level at 40 : a study of state-of-theart use, challenges, and opportunities", In PICMET 15: Management of the technology age. pp. 2084-2094. http://dx.doi.org/10.1109/picmet.2015.7273196

[10] Chad L. Dacus, "Improving acquisition outcomes through simple system technology readiness metrics," Defense ARJ, vol. 19, no. 4, pp. 444-461, 2012.

[11] B. Sauser., J. Ramirez-Marquez and D. Henry, "A system maturity index for the systems engineering life cycle", Int. J. Industrial and Systems Engineering, 3 (6), 2008, pp. 673691

[12] M. Farhana, E. Bimenyimana, "Design Driven Innovation as a Differentiation Strategy- In the Context of Automotive Industry", J. Technol., Manag. Innov. vol 15, No 2, 2015, pp. 24-38.

[13] John A. Burgess, Design Assurance for Engineers and Managers. CRC press, 1984, pp 162-180. \{ISBN: 9781441-94260-9\}

[14] Kristina Launche, "Sketching a strategy: early design in different industrial sectors," in Proc. International Conference on Engineering Design Conf., ICED03, (Stockholm; 19-21 August 2003), 10 pp., 2003. 
Proc. 2017 Canadian Engineering Education Association (CEEA17) Conf.

CEEA17; Paper 085

University of Toronto; June 4-7, 2017

-4 of $4-$ 